\title{
A novel bone cement screw system combined with vertebroplasty for the treatment of Kummell disease with bone deficiency at vertebral anterior border: minimum 3-year follow-up study
}

\author{
Biao Wang \\ Honghui Hospital \\ Xinliang Zhang \\ Honghui Hospital \\ Lingbo Kong \\ Honghui Hospital \\ Li Yuan \\ Honghui Hospital \\ Simin He \\ Honghui Hospital \\ Liang Yan \\ Honghui Hospital
}

Dingjun Hao ( $\nabla$ dingjunhaowb@163.com )

Honghui Hospital, Xi'an Jiaotong University

Research article

Keywords: Kummell disease, vertebroplasty, bone cement screw, cement displacement

Posted Date: March 27th, 2020

DOI: https://doi.org/10.21203/rs.3.rs-18781/v1

License: (a) (i) This work is licensed under a Creative Commons Attribution 4.0 International License. Read Full License

Version of Record: A version of this preprint was published at Clinical Neurology and Neurosurgery on February 1st, 2021. See the published version at https://doi.org/10.1016/j.clineuro.2020.106434. 


\section{Abstract}

Background: When vertebroplasty is used to treat Kummell disease with bone deficiency at vertebral anterior border, bone cement displacement often occurs intraoperative or postoperative. We designed and used a new bone cement screw system to avoid the serious complication. The purpose of this study is to evaluate the safety and effectiveness of this novel operation method through more than 3 years of follow-up.

Methods: From January 2012 to August 2016, 27 patients suffering from single-segment Kummell disease with bone deficiency at vertebral anterior border were treated by vertebroplasty combined with novel bone cement screw. Bone cement is released into the diseased vertebrae through screw to fully fill the intravertebral vacuum cleft. Screw fixation of bone cement can avoid intraoperative or postoperative displacement. All patients were operated by unilateral technique, only one screw was implanted for each patient. The clinical efficacy was evaluated using Odom's criteria and statistical analysis based on the results of vertebral body index (VBI), vertebral body angle (VBA), bisegmental Cobb angle (BCA), visual analogue scale (VAS), oswestry disability index (ODI), and the MOS 36-item short from health survey (SF36).

Results: The operation was completed successfully in 27 cases. The average operation time was $49.63 \pm 10.82 \mathrm{~min}$, and the average volume of cement injected was $4.70 \pm 0.87 \mathrm{ml}$. The patients' preoperative $\mathrm{VBI}, \mathrm{VBA}, \mathrm{BCA}$, VAS and ODI scores were $43.11 \pm 5.94,21.04 \pm 2.55,45.00 \pm 6.26,7.59 \pm 0.84$, and $79.85 \pm 7.58$, respectively. The postoperative measurements were $78.70 \pm 2.55,12.70 \pm 2.11,26.11 \pm 4.73$, $3.22 \pm 0.93$ and $50.04 \pm 9.28$. At the last follow-up, the measurements were $78.04 \pm 2.30,13.15 \pm 2.38$, $27.07 \pm 4.87,2.04 \pm 0.65$, and $22.85 \pm 5.06$, respectively. There was significant difference between the preoperative and postoperative data, as well as the preoperative and the last follow-up data $(P<0.05)$. Compared the results of SF-36 preoperative and at the last follow-up, there were significant differences in physical function, role-physical, body pain, vitality, and social function these 5 items $(P<0.05)$. However, there were no significant differences in general health, emotional function and mental health. Finally, 26 patients (96.3\%) had good to excellent clinical outcomes according to Odom's criteria.

Conclusions: This 3-year follow-up study shows that the novel bone cement screw system combined with vertebroplasty has a good short and medium-term therapeutic effect on patients with Kummell disease and bone deficiency at vertebral anterior border, while its long-term efficacy is subject to further studies.

\section{Introduction}

Delayed posttraumatic spinal vertebral body collapse, namely Kummell disease, first described by Dr. Hermann Kummell in 1985 is a rare and poorly documented disease [1, 2]. By definition, it is a spinal disorder characterized by patients suffered a minor spinal trauma but resulted in a symptomatic, progressive, angular kyphosis after a symptom-free period of time ranging from months to years $[3,4]$. 
For this rarely seen disease, the treatments are tricky and no consolidate method $[2,5]$. The goal of treatment is to relieve the back pain, and to prevent the affected vertebra from further collapse, and hence prevent kyphotic progressing [6]. Conservative management such as bed rest, narcotic analgesics, and use of a brace often use as an initial treatment for Kummell disease. However, patients often turn to surgical intervention because of poor treatment efficacy.

Nowadays, traditional open internal instrumentation and fusion are used to face the challenge. Over the past decade, the techniques for minimally invasive spinal surgery have improved significantly [7-10]. Percutaneous vertebroplasty has become the main treatment approach for Kummell disease due to it can achieve pain relief and satisfactory deformity correction [11-13]. However, for Kummell disease patients with severe intravertebral instability, especially those who are confirmed by preoperative computed tomography (CT) with bone deficiency due to gasification or liquefaction at vertebral anterior border, catastrophic complications such as intraoperative or postoperative cement displacement often occur (Fig. 1) [14-18]. Once bone cement displacement occurs, patients usually require undergoing revision surgery via open posterior approach, anterior approach or even a combination of posterior and anterior approaches. Nevertheless, such kinds of revision surgery are not only difficult and risky, but also are intolerable for elderly patients, which often makes the treatment very tricky.

In order to avoid the extremely serious complication of bone cement displacement, we have developed a novel type of percutaneous bone cement fixation screw system for vertebroplasty in the treatment of Kummell disease with bone deficiency at vertebral anterior border. In the present study, we retrospectively analyzed 27 patients with Kummell disease and bone deficiency at vertebral anterior border who were treated with this novel screw system in combination with vertebroplasty.

\section{Methods}

\section{General information}

Between July 2012 and August 2016, a total of 27 patients with single-segment Kummell disease (27 vertebral bodies) with bone deficiency at vertebral anterior border confirmed by CT and magnetic resonance imaging (MRI) were selected. They underwent vertebroplasty treatment combined with a novel percutaneous cement fixation screw system, including 6 males and 21 females, age of 69-88 years (mean age of $75.19 \pm 4.91$ years). The preoperative bone density $\mathrm{T}$ score measured by double $\mathrm{X}$-ray absorptiometry ranged $-2.9 \sim-5.5$, with an average of $-3.58 \pm 0.59$. In terms of disease location, there was 1 case of T10, 3 cases of T11, 15 cases of T12 and 8 cases of L1. Three cases were accompanied by diabetes, 8 cases by hypertension and 7 cases by coronary heart disease. Li classification [19] was adopted for staging, which indicated 20 cases of stage II and 7 cases of stage III (7 patients had spinal cord compression but no neurological symptoms). After an explanation of the surgical options, all patients signed an informed consent form authorized by the local ethics committee. The patients' demographic information was given in table 1 . 


\section{Inclusion and exclusion criteria}

Inclusion criteria: single-segment Kummell disease with bone deficiency at vertebral anterior border, bone density $T$ score was smaller than -2.5 , with severe back pain and no neurological deficit.

Exclusion criteria: metastatic spine tumors, multilevel Kummell lesions, long-term use of anti-coagulant drugs, senile dementia, cognitive damage or other cerebral disease, malignant disease, spinal infection or skin disease.

\section{Surgical methods}

All patients underwent surgery after preoperative examination and anesthesia evaluation. After general anesthesia, the patients were placed in a prone position, with vacant abdomen by padding the chest and anterior superior iliac spine using a soft pad, followed by adjusting the surgical bed under the C-arm fluoroscopy to position the patient in the appropriate overextending position to optimize the restoration of the vertebral height. Then the surface projections of the bilateral pedicle margin of the diseased vertebra were marked under the $\mathrm{C}$-arm fluoroscopy. All the 27 patients underwent surgery via unilateral surgical approach, in which the side with the more severe vertebral cleft on CT was selected. According to the patient's somatotype, the skin was cut (length about $10 \mathrm{~mm}$ ) longitudinally 5-10 mm outside the surface marker, and then the subcutaneous tissue and the thoracolumbalis fascia were cut layer by layer. Subsequently, under the guidance of anteroposterior fluoroscopy, the needle tip was placed on the outer edge of the pedicle projection (10 o'clock position on the left and 2 o'clock position on the right), and inclined $10 \sim 15^{\circ}$ inward to puncture the vertebral cleft. After the needle tip was found reaching the rear edge of the vertebra under the guidance of lateral fluoroscopy, it was confirmed without breaking the medial edge of the pedicle projection (to avoid the screw from entering the spinal canal as much as possible) under the guidance of anterior and posterior fluoroscopy. Subsequently, the needle further punctured to the vertebral fissure with the guidance of lateral fluoroscopy, and the guide wire was inserted. The guide wire should not be excessively inserted to avoid breaking the vertebra during screw insertion, so as to avoid damaging ventral blood vessels and other viscera. Then the novel cement screw with bone cement outlet (Fig. 2) was placed percutaneously into the vertebral cavity under the guidance of the guide wire. The bone cement injection device was connected to the end of the needle. Then, under the lateral X-ray fluoroscopy, an appropriate volume (usually about 3-6 $\mathrm{ml}$ ) of bone cement in the drawing or agglomeration phase was slowly injected until the cleft in the diseased vertebra was fully filled. After the bone cement was solidified, the bone cement injection device was removed and the wound was sutured. The schematic diagram of the operation was shown in Fig. 3.

\section{Postoperative management}

Antibiotics were routinely applied for 24 hours after surgery. On the first postoperative day, patients were allowed to ambulate under the protection of the thoracolumbar brace, and were discharged after the conditions were stabilized. After discharge from the hospital, they continued to wear brace for 12 weeks, 
and took calcium, vitamin D3 and Bisphosphonates for anti-osteoporosis treatment under the guidance of doctors from osteoporosis department.

\section{Efficacy evaluation}

Patients were reviewed $3,6,12,24,36$ months after the operation, during which X-ray and CT were used to evaluate the presence of bone cement displacement, progress of kyphosis, new onset compression fracture, as well as presence of loosening, displacement of the cement screws, etc. Kyphotic deformity and radiographic follow-up were defined by the vertebral body index (VBI: relation between the anterior and posterior wall height of the diseased vertebra), vertebral body angle (VBA: angle defined by the upper and lower endplate of the diseased vertebra), and bisegmental Cobb angle (BCA: angle defined by the upper endplate of the first vertebra above the diseased one and by the lower endplate of the first vertebra below the diseased one). The measurement method is shown in Fig. 4.

Patients were assessed for pain relief and functional recovery by comparing Visual Analogue Scale (VAS, 0: no pain at all; 10: worst pain imaginable) and Oswestry Disability Index (ODI, 0\%: best functional state; $100 \%$ : worst functional state) scores.

The general health status of the patients was evaluated according to the MOS 36-item short from health survey (SF-36) completed before operation and at the last follow-up. The improvement of eight dimensions: physical function (PF), role-physical (RP), body pain (BP), general health (GH), vitality (VT), social function (SF), emotional function (RE) and mental health $(\mathrm{MH})$ were evaluated respectively.

Odom's criteria were used to evaluate the clinical satisfaction of the 27 patients at the most recent followup (It is divided into four levels: excellent, good, satisfaction, poor; excellent is the best and poor is the worst).

\section{Statistical analysis}

Statistical analyses were performed using SPSS 21.0 software. The VBI, VBA, BCA, VAS, and ODI scores before surgery, after surgery and at the end of the 3-year follow-up were compared using Paired t-test. Changes of SF-36 eight dimensions scores before surgery and at the last follow-up were also compared using Paired t-test. All the data were expressed as mean \pm standard deviation. A difference with $P<0.05$ was considered statistically significant.

\section{Results}

\section{Clinical results}

The operation was completed successfully in 27 patients. A total of 27 screws were placed. Postoperative CT examination showed that all the screws were in good position, and no bone cement displacement occurred during the operation. The average operation time was 49.63 $\pm 10.82 \mathrm{~min}$ (ranged 30-70 min). The average volume of cement injected was $4.70 \pm 0.87 \mathrm{ml}$ (ranged $3-6 \mathrm{ml}$ ). Blood loss during the 
operation was minimal. During operation, 4 cases (14.81\%) occurred bone cement leakage, of which 3 cases were lateral leakage of vertebral body, and 1 case was leakage of superior disc. No case had venous leakage. None of the patients suffered from serious complications such as injury of large blood vessels or spinal cord, etc. Wound was well healed in all patients, with absence of delayed wound healing, infection and hematoma. The average time of hospital stays were $3.30 \pm 0.95$ days (ranged 2-5 days). The clinical results were shown in table 1.

\section{Imaging and follow-up results}

During the 3-year follow-up, no patients had complications such as loosening, displacement, and fracture of cement screws, and no cases had delayed cement displacement. In addition, during the follow-up, none of the patients complained of the skin or soft tissue wear discomfort caused by the screws. One patient suffered from a new fracture at non-adjacent segment of thoracolumbar vertebra (new fracture rate: $3.70 \%$ ). The patient's fractured vertebral height was not significantly lost, with tolerable pain, and gained rehabilitation after conservative treatment. Compared the imaging parameters obtained before surgery, after surgery and at the end of the 3-year follow-up, it was found that the VBI, VBA, and BCA scores were significantly improved after treatment using the novel percutaneous cement screw system combined with vertebroplasty. The VBI was increased from $43.11 \pm 5.94$ before surgery to $78.70 \pm 2.55$ after surgery, and was $78.04 \pm 2.30$ at the end of the 3-year follow-up. VBA was decreased from $21.04 \pm$ 2.55 before surgery to $12.70 \pm 2.11$ after surgery, and was $13.15 \pm 2.38$ at the end of follow-up. BCA was improved from $45.00 \pm 6.26$ before surgery to $26.11 \pm 4.73$ after surgery, and was $27.07 \pm 4.87$ at the end of the 3-year follow-up. In addition, there were significant differences between these three parameters obtained preoperatively, postoperatively and at the final follow-up $(p<0.05)$. This indicates that this novel treatment method can well restore the height and improve kyphosis of vertebra with Kummell disease. Although the imaging parameters were slightly decreased at the end of 3-year follow-up, there were no significant differences between these parameters obtained in the last follow-up and after surgery ( $p$ > 0.05), which indicates that the height of the vertebral body and the curvature of thoracolumbar spine are well maintained after surgery. The statistical results were shown in Table 2.

At the last follow-up, the VAS and ODI scored $2.04 \pm 0.65$ and $22.85 \pm 5.06$, respectively, which were significantly improved compared with those of $7.59 \pm 0.84$ and $79.85 \pm 7.58$, respectively before surgery $(P<0.05)$. The improvement rates of VAS and ODI were respectively $73.12 \%$ and $71.38 \%$ [improvement rate $=($ final follow-up score - preoperative score) $/$ (score at the best status - preoperative score) $* 100 \%]$. The statistical results were shown in Table 2.

All 27 patients completed SF-36 scale evaluation; the physical function (PF) score was increased from $45.19 \pm 6.28$ before surgery to $77.96 \pm 6.69$ at the last follow-up. The role-physical (RP) score was increased from $28.70 \pm 19.25$ before surgery to $75.00 \pm 16.98$ at the final follow-up. The Body pain (BP) score was increased from $15.93 \pm 8.62$ before surgery to $68.15 \pm 6.18$ at the final follow-up. The Vitality (VT) score was increased from $48.33 \pm 11.68$ before surgery to $62.04 \pm 11.37$ at the final follow-up. The Social function (SF) score was increased from $29.17 \pm 10.40$ before surgery to $34.72 \pm 11.14$ at the final follow-up. After statistical analysis of t-test, there were significant differences in PF, RP, BP, VT, and SF 
these 5 items compared before operation and at the last follow-up $(P<0.05)$. However, there were no significant differences in general health (GH), emotional function (RE) and mental health (MH) these 3 items compared before operation and at the last follow-up $(P>0.05)$. The statistical results were shown in Table 3.

Finally, according to Odom's criteria, 26 patients (96.3\%) had good to excellent clinical outcomes in the aggregate. 19 patients $(70.4 \%)$ had excellent results, 7 patients $(25.9 \%)$ had good results, 1 patient $(3.7 \%)$ had satisfactory result, and no patient had poor result, respectively (Table 4). A typical case was shown in Fig. 5.

\section{Discussion}

With the aging of the global population, the number of patients with spinal fractures, especially osteoporotic spinal fractures, has increased year by year [20, 21], and patients with Kummell disease have also increased clinically. If one patient without manifests asymptomatic for a few months or even a few years after experiencing mild trauma, and then has pain in the same site, with worsened symptoms, which gradually progresses to kyphosis, and even appears neurological symptoms, it is referred to posttraumatic vertebral delayed osteonecrosis or Kummell disease. The disease often occurs in the elderly, more common in the thoracolumbar spine, the most common spinal segment is the T12 vertebra, male to female ratio of about $1: 10[22,23]$.

Intravertebral vacuum cleft (IVC) is a characteristic imaging manifestation of Kummell disease. Libicher et al [24] found that IVC showed a sensitivity of up to $85 \%$ and a specificity of $99 \%$ in the diagnosis of Kummell disease. However, IVC is not the basis for the diagnosis of Kummell disease. It can be not only found in Kummell disease, but also found in acute fracture, infection, primary neoplasm or metastasis etc $[2,25]$. IVC is formed by gas or liquid accumulation in the vertebral body. It is usually located in the middle of the vertebral bodies or adjacent to the compression endplate in the coronal view, and is most commonly located in the anterior edge of the vertebral body in the sagittal view [26]. Hence, for Kummell disease, bone deficiency in the anterior edge of vertebral body is very common clinically.

Currently, the pathogenesis of Kummell disease is still unclear. In 1951, Steel et al. [27] first proposed a possible mechanism of formation of cancellous bone fracture and small hematoma caused by tiny spinal trauma, which further led to osteonecrosis and eventually collapse of the vertebral body. With the deepening of research in recent years, hypotheses of pseudo-arthrosis and ischemic necrosis in the vertebral body have become the mainstream. Kim et al. [28] believed that IVC indicated the existence of dynamic activities of non-union pseudo-articular joints in the micro-fracture end, which further resulted in osteonecrosis and vertebral collapse, rather than ischemic necrosis. Furthermore, biopsies of the IVC demonstrated fibrocartilage within a fibrous stroma, findings that represent a pseudo-synovium of a pseudarthrosis. This false joint is indicative of a permanent failure of bone healing after fracture $[29,30]$. However, more scholars favor that Kummell disease is caused by ischemic necrosis of the vertebral body. Yu et al. [26] and Matzaroglou et al. [31] performed biopsy of Kummell diseased vertebral bodies, and 
found trabecular ischemic necrosis. All of these results supported the hypothesis of vertebral ischemic necrosis.

With the rapid development of minimally invasive spine surgery, vertebroplasty and kyphoplasty have become the main methods for the treatment of Kummell disease without neurological damage due to advantages such as small trauma, good tolerance, rapid pain relief and effective deformity correction, etc $[3,11-13,32,33]$. In theory, kyphoplasty is superior to vertebroplasty, with a better vertebral height reduction and a lower probability of bone cement leakage. However, for Kummell disease, especially for phases II and III patients, the most important aspect of vertebral height restoration is to use the overextending position to fully open IVC instead of reduction using a balloon. At the same time, when the bone cement is targeted and injected into the IVC, bone cement leakage is unlikely to occur since the IVC walls are composed of hardened bone, except that it is prone to occur anterior bone cement leakage in patients with anterior vertebral body deficiency. Krauss et al. [32] implemented vertebroplasty in 44 cases with osteoporotic fractures plus IVC and 148 cases with osteoporotic fractures without IVC, and found that the bone cement leakage rate was much lower in the patients with IVC. In addition, for Kummell disease, the use of a balloon may rupture the thin endplate, and result in intervertebral leakage of bone cement, and thus increase the probability of adjacent vertebral fractures. Therefore, in clinical practice, vertebroplasty is more commonly used instead of kyphoplasty after the overextending position, which is also more economical.

Although the use of bone cement has achieved satisfactory clinical results [3,33,34], the catastrophic complication of bone cement displacement during or after surgery is inevitably worrying. Even if the postoperative results are satisfactory, there is still concern of bone cement displacement during followup. Once bone cement displacement occurs, patients often need to undergo open posterior, anterior, or even anterior and posterior revision surgery to remove the displaced bone cement, reconstruct spinal stability, restore spinal sequence and fusion $[16,17,35]$. Thus, some scholars intended to apply short or long segmental fixation combined with vertebroplasty to avoid bone cement displacement. However, compared with using the bone cement alone, it has the disadvantages of large surgical trauma, loss of spinal mobility and high cost $[3,36]$.

The reason for postoperative bone cement displacement for Kummell disease is still unclear. We summarized and analyzed the Kummell disease patients with bone cement displacement after VP or KP who were treated in our center or reported in nearly all literature in recent years $[14-18,35,37]$, and found that all these patients suffered from bone deficiency at vertebral anterior border, and the bone cement shifted from the front of the deficient vertebra to ventral part. In the vertebroplasty, the bone cement tends to distribute on the vertebral anterior border under the action of the push pressure, but is easy to leak into the front of the vertebral body under pressure in case of bone deficiency at vertebral anterior border. In the meantime, even with perfect postoperative kyphosis correction, the patients may still be in mild kyphosis compared with the status before the disease onset. Hence, in postoperative activity, the barycenter of the diseased vertebra is moved forward, and the stress can squeeze the bone cement to produce a force for anterior displacement, thereby results in anterior displacement of bone cement since there is no bone 
barrier due to bone deficiency at vertebral anterior border. Wang et al. [15] and Tsai et al. [16] also believed that the integrity of the anterior cortex is an important reason for anterior displacement of bone cement. In addition, the permeation of bone cement into trabecular bone plays an important role in preventing leakage and displacement, both before and after the procedure [38]. It has been confirmed by pathology that IVC is a dead space composed of necrotic bone tissue. After filling with bone cement, it is difficult for the bone cement to infiltrate into the normal trabecular structure, meaning that the bone cement and the surrounding bone tissue are non-union, which is another important reason for cement dislodgment [17, 18]. Tsai et al. [16] believed PMMA cement in vertebroplasty merely a space occupying material without mechanical interlock and biocompatibility and, thus, make the potential for dislodgment.

In order to interlock the bone cement and the surrounding bone tissue to avoid bone cement displacement during or after surgery, we have developed a novel bone cement screw system. The key technology is that after the bone cement screw is inserted in the IVC, the bone cement will slowly release through the lateral hole at the front end of the screw and completely fill the IVC during injection, which tends to integrate the bone cement and the screw, while maintaining the screw in the vertebral body. Hence, the cement screw acts as a "bridge" to interlock the bone cement with the surrounding bone tissue, and even makes it connect to the pedicle that is the most rigid part, which strengthens the linkage, and the screws are unlikely to loosen and shift as well. Furthermore, the tail-free design of the screws allows the bone cement screw to completely locate in the bone structure, which is unlikely to wear the muscles and other soft tissue during the activity while acting as a bridge. At the end of the 3-year follow-up, none of the 27patients had bone cement displacement and screw loosening, with a VAS improvement rate of $73.12 \%$ and ODI improvement rate of $71.38 \%$. These preliminary results reveal that the screw system is safe and effective, and plays a good short and medium-term therapeutic effect on patients with Kummell disease and bone deficiency at vertebral anterior border, while its long-term efficacy needs further observation. In addition, the development of a screw system that enables the growth of bone tissue in it may provide an even better stability.

\section{Conclusions}

The 3-year follow-up of the 27 patients shows that the novel bone cement screw system combined with vertebroplasty has a good short and medium-term therapeutic effect on patients with Kummell disease and bone deficiency at vertebral anterior border, while its long-term efficacy is subject to further studies.

\section{Declarations}

\section{Acknowledgements}

We are thankful for the support of the nursing staffs from the Department of Spine Surgery, Xi'an Jiaotong University College of Medicine, Honghui Hospital.

\section{Funding}


This work was supported by the National Natural Science Foundation of China (No.81802167), Natural Science Research Plan in Shaanxi Province of China (No.2019JM197), and Medical Research Project of Xi'an Science and Technology Bureau (201805096YX4SF30-4).

\section{Availability of data and materials}

The datasets used and analysed during the current study are available from the corresponding author on reasonable request.

\section{Authors' contributions}

Dingjun Hao and Biao Wang designed the research, analyzed the data and wrote the manuscript. Xinliang Zhang, Li Yuan, and Lingbo Kong acquired data. Simin He, Liang Yan, and Biao Wang performed surgical treatment. Dingjun Hao supervised the project and reviewed the manuscript.

\section{Ethics approval and consent to participate}

This study was approved by the Medical Ethics Committee of Honghui Hospital and informed consent was obtained from all of the individual participants included in the study.

\section{Consent for publication}

Written informed consent for publication was obtained from all patients.

\section{Competing interests}

The authors declare that they have no competing interests.

\section{References}

1. Lim J, Choi SW, Youm JY, Kwon HJ, Kim SH, Koh HS (2018) Posttraumatic Delayed Vertebral Collapse : Kummell's Disease. J Korean Neurosurg Soc 61:1-9

2. Matzaroglou C, Georgiou CS, Assimakopoulos K, Giannakenas C, Karageorgos A, Saridis A et al (2011) Kümmell's disease: pathophysiology, diagnosis, treatment and the role of nuclear medicine. Rationale according to our experience. Hell J Nucl Med 4:291-299

3. Chen L, Dong R, Gu Y, Feng Y (2015) Comparison between Balloon Kyphoplastyand Short Segmental Fixation Combined with Vertebroplasty in the Treatment of Kümmell's Disease. Pain Physician 18:373-381

4. Masala S, Nano G, Mammucari M, Simonetti G (2011) Kummel Disease Treatment by Unipedicular Vertebral Augmentation Using Curved Injection Cannula. Cardiovasc Intervent Radiol 34:1014-1020

5. Lee SH, Kim ES, Eoh W (2011) Cement augmented anterior reconstruction with short posterior instrumentation: a less invasive surgical option for Kummell's disease with cord compression. J Clin Neurosci 18:509-514 
6. Yang H, Pan J, Wang G (2014) A Review of Osteoporotic Vertebral Fracture Nonunion Management. Spine (Phila Pa 1976) 39:B4-6

7. Zhao Q, Hao D, Wang B (2018) A novel, percutaneous, self-expanding, forceful reduction screw system for the treatment of thoracolumbar fracture with severe vertebral height loss. J Orthop Surg Res 13:174.

8. Koreckij T, Park DK, Fischgrund J (2014) Minimally invasive spine surgery in the treatment of thoracolumbar and lumbar spine trauma. Neurosurg Focus 37:E11

9. Wang B, Guo H, Yuan L, Huang D, Zhang H, Hao D (2016) A prospective randomized controlled study comparing the pain relief in patients with osteoporotic vertebral compression fractures with the use of vertebroplasty or facet blocking. Eur Spine J 25:3486-3494

10. Snyder LA, O'Toole J, Eichholz KM, Perez-Cruet MJ, Fessler R (2014) The technological development of minimally invasive spine surgery. Biomed Res Int 2014:293582

11. Peh WC, Gelbart MS, Gilula LA, Peck DD (2003) Percutaneous vertebroplasty: treatment of painful vertebral compression fractures with intraosseous vacuum phenomena. AJR Am J Roentgenol 180:1411e1417.

12. Ma R, Chow R, Shen FH (2010) Kummell's disease: delayed post-traumatic osteonecrosis of the vertebral body. Eur Spine J 19:1065-1070

13. Liu F, Chen Z, Lou C, Yu W, Zheng L, He D et al (2018) Anterior reconstruction versus posterior osteotomy in treating Kümmell's disease with neurological deficits: A systematic review. Acta Orthop Traumatol Turc 52:283-288

14. Kim JE, Choi SS, Lee MK, Lee DK, Cho SI (2017) Failed Percutaneous Vertebroplasty Due to Insufficient Correction of Intravertebral Instability in Kummell's Disease: A Case Report. Pain Pract 17:1109-1114

15. Wang HS, Kim HS, Ju Cl, Kim SW (2008) Delayed bone cement displacement following balloon kyphoplasty. J Korean Neurosurg Soc 43:212-214

16. Tsai TT, Chen WJ, Lai PL, Chen LH, Niu CC, Fu TS et al (2003) Polymethylmethacrylate cement dislodgment following percutaneous vertebroplasty: a case report. Spine (Phila Pa 1976) 28:E457E460.

17. Nagad P, Rawall S, Kundnani V, Mohan K, Patil SS, Nene A (2012) Postvertebroplasty instability. J Neurosurg Spine 16:387-393

18. Jeong YH, Lee CJ, Yeon JT, Bae J, Choi E, Lee PB et al (2016) Insufficient Penetration of Bone Cement Into the Trabecular Bone: A Potential Risk for Delayed Bone Cement Displacement After Kyphoplasty? Reg Anesth Pain Med 41:616-618

19. Li KC, Li AF, Hsieh CH, Liao TH, Chen CH (2007) Another option to treat Kümmell's disease with cord compression. Eur Spine J 16:1479-1487

20. Cui L, Chen L, Xia W, Jiang Y, Cui L, Huang W et al (2017) Vertebral fracture in postmenopausal Chinese women: a population-based study. Osteoporos Int 28:2583-2590 
21. Rajasekaran S, Kanna RM, Schnake KJ, Vaccaro AR, Schroeder GD, Sadiqi S et al (2017) Osteoporotic Thoracolumbar Fractures-How Are They Different?-Classification and Treatment Algorithm. J Orthop Trauma 31:S49-S56

22. Osterhouse MD, Kettner NW (2002) Delayed posttraumatic vertebral collapse with intravertebral vacuum cleft. J Manipulative Physiol Ther 25:270-275

23. Young WF, Brown D, Kendler A, Clements D (2002) Delayed post-traumatic osteonecrosis of a vertebral body (Kummell's disease). Acta Orthop Belg 68:13-19

24. Libicher M, Appelt A, Berger I, Baier M, Meeder PJ, Grafe I et al (2007) The intravertebral vacuum phenomen as specific sign of osteonecrosis in vertebral compression fractures: results from a radiological and histological study. Eur Radiol 17:2248-2252

25. Feng SW, Chang MC, Wu HT, Yu JK, Wang ST, Liu CL (2011) Are intravertebral vacuum phenomena benign lesions? Eur Spine J 20:1341-1348

26. Yu CW, Hsu CY, Shih TT, Chen BB, Fu CJ (2007) Vertebral osteonecrosis: MR imaging findings and related changes on adjacent levels. AJNR Am J Neuroradiol 28:42-47

27. STEEL HH (1951) Kümmell's disease. Am J Surg 81:161-167

28. Kim DY, Lee SH, Jang JS, Chung SK, Lee HY (2004) Intravertebral vacuum phenomenon in osteoporotic compression fracture: report of 67 cases with quantitative evaluation of intravertebral instability. J Neurosurg 100:24-31

29. Hasegawa K, Homma T, Uchiyama S, Takahashi H (1998) Vertebral pseudarthrosis in the osteoporotic spine. Spine (Phila Pa 1976) 23: 2201-2206

30. Hasegawa K, Homma T, Uchiyama S, Takahashi HE (1997) Osteosynthesis without instrumentation for vertebral pseudarthrosis in the osteoporotic spine. J Bone Joint Surg Br 79: 452-456

31. Matzaroglou C, Georgiou CS, Assimakopoulos K, Saridis A, Khudiaev AT, Giannakenas C (2010) Kümmell' s disease: A rare spine entity in a young adult. Hell J Nucl Med 13:52-55

32. Krauss M, Hirschfelder H, Tomandl B, Lichti G, Bär I (2006) Kyphosis reduction and the rate of cement leaks after vertebroplasty of intravertebral clefts. Eur Radiol 16:1015-1021

33. Chen GD, Lu Q, Wang GL, Zou J, Yang HL, Yang Y et al (2015) Percutaneous Kyphoplasty for Kummell Disease with Severe Spinal Canal Stenosis. Pain Physician 18:E1021-E1028

34. Zhang J, Fan Y, He X, Meng Y, Huang Y, Jia S et al (2018) Is percutaneous kyphoplasty the better choice for minimally invasive treatment of neurologically intact osteoporotic Kümmell's disease? A comparison of two minimally invasive procedures. Int Orthop 42:1321-1326

35. Zhang C, Wang G, Liu X1, Li Y, Sun J (2017) Failed percutaneous kyphoplasty in treatment of stage 3 Kummell disease: A case report and literature review. Medicine (Baltimore) 96:e8895

36. Li HK, Hao DJ, Yang JS, Huang DG, Yu CC, Zhang JN et al (2017) Percutaneous kyphoplasty versus posterior spinal fixation with vertebroplasty for treatment of Kümmell disease: A case-control study with minimal 2-year follow-up. Medicine (Baltimore) 96:e9287 
37. Wagner AL, Baskurt E (2006) Refracture with cement extrusion following percutaneous vertebroplasty of a large interbody cleft. AJNR Am J Neuroradiol 27:230-231

38. Phillips FM, Todd Wetzel F, Lieberman I, Campbell-Hupp M (2002) An in vivo comparison of the potential for extravertebral cement leak after vertebroplasty and kyphoplasty. Spine (Phila Pa 1976) 27:2173-2178

\section{Tables}

Due to technical limitations, the tables are only available as a download in the supplemental files section.

\section{Figures}

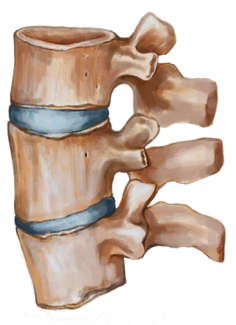

A

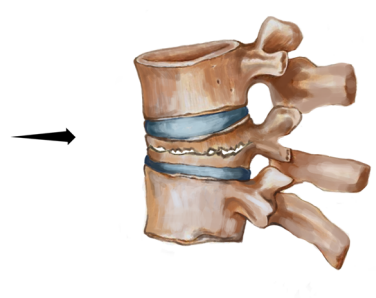

B

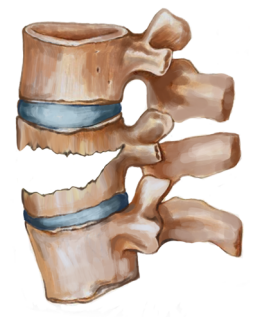

C

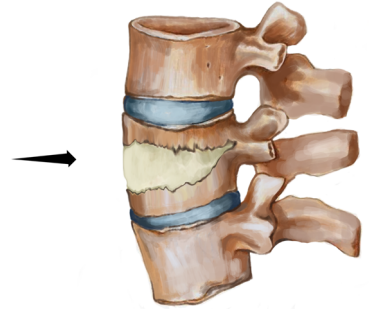

D

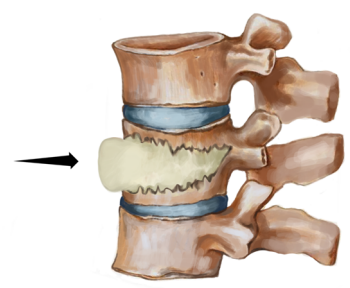

E

\section{Figure 1}

Schematic diagram of PVP treatment and postoperative cement displacement in Kummell disease. A: normal vertebral body; B: Kummell disease causes vertebral collapse and kyphosis; C: After reduction of collapsed vertebrae and correction of spinal curvature with hyperextension, bone deficiency was found at the anterior border of vertebrae; D: use bone cement to fill the bone defect site and complete PVP treatment; E: Postoperative bone cement displacement and loss of spinal curvature correction. 


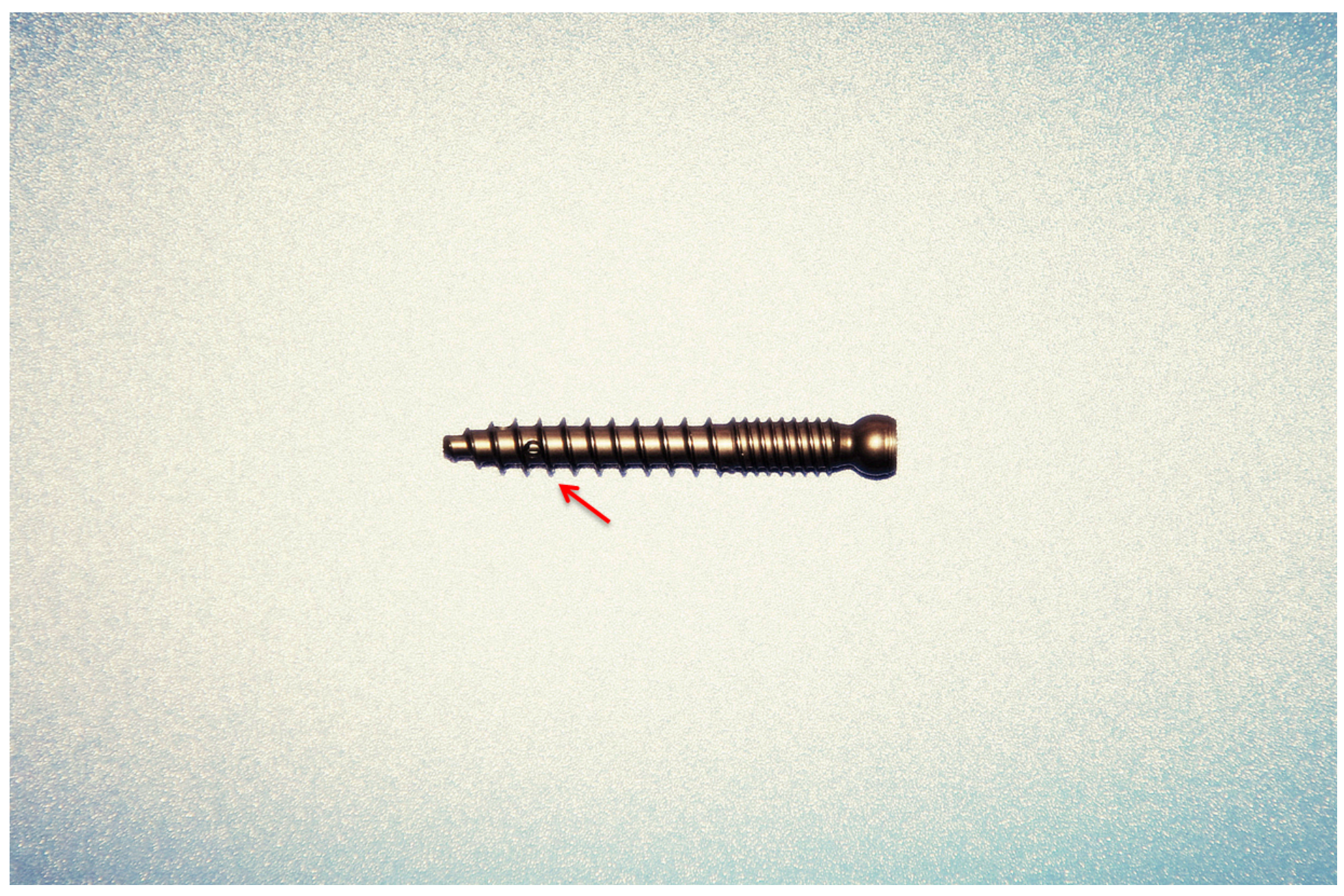

Figure 2

Schematic diagram of new bone cement screw. There are 4 cement outlets with different distribution at the front of the screw, as shown by the red arrow.

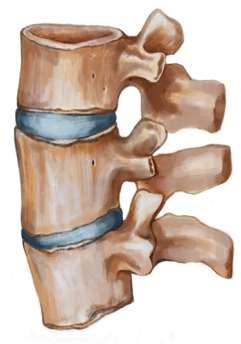

A
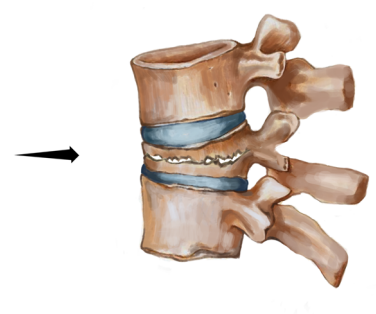

B
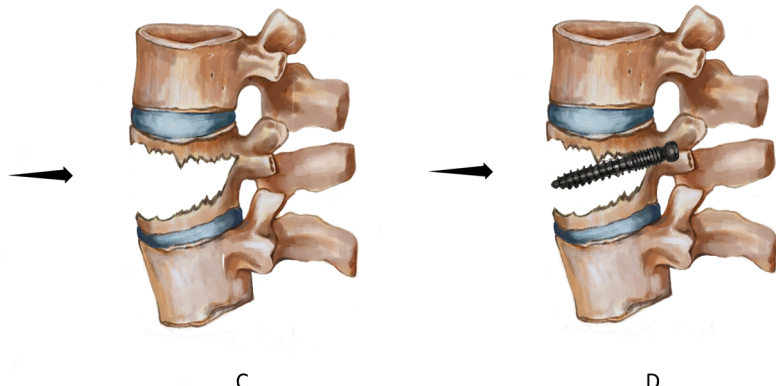

D

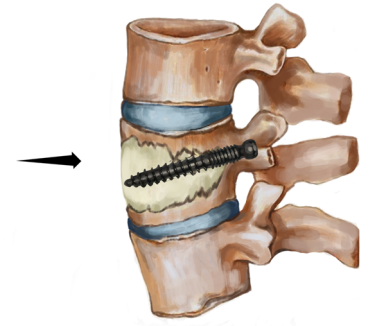

E

Figure 3 
Schematic diagram of Kummell disease treated by new bone cement screw system combined with PVP. A: normal vertebral body; B: Kummell disease causes vertebral collapse and kyphosis; C: After reduction of collapsed vertebrae and correction of spinal curvature with hyperextension, bone deficiency was found at the anterior border of vertebrae; D: percutaneous minimally invasive implantation of new bone cement screw in the intravertebral vacuum cleft; E: PVP treatment is completed by fully filling bone cement through new bone cement screw into the cleft, and the displacement of bone cement is avoided by screw.

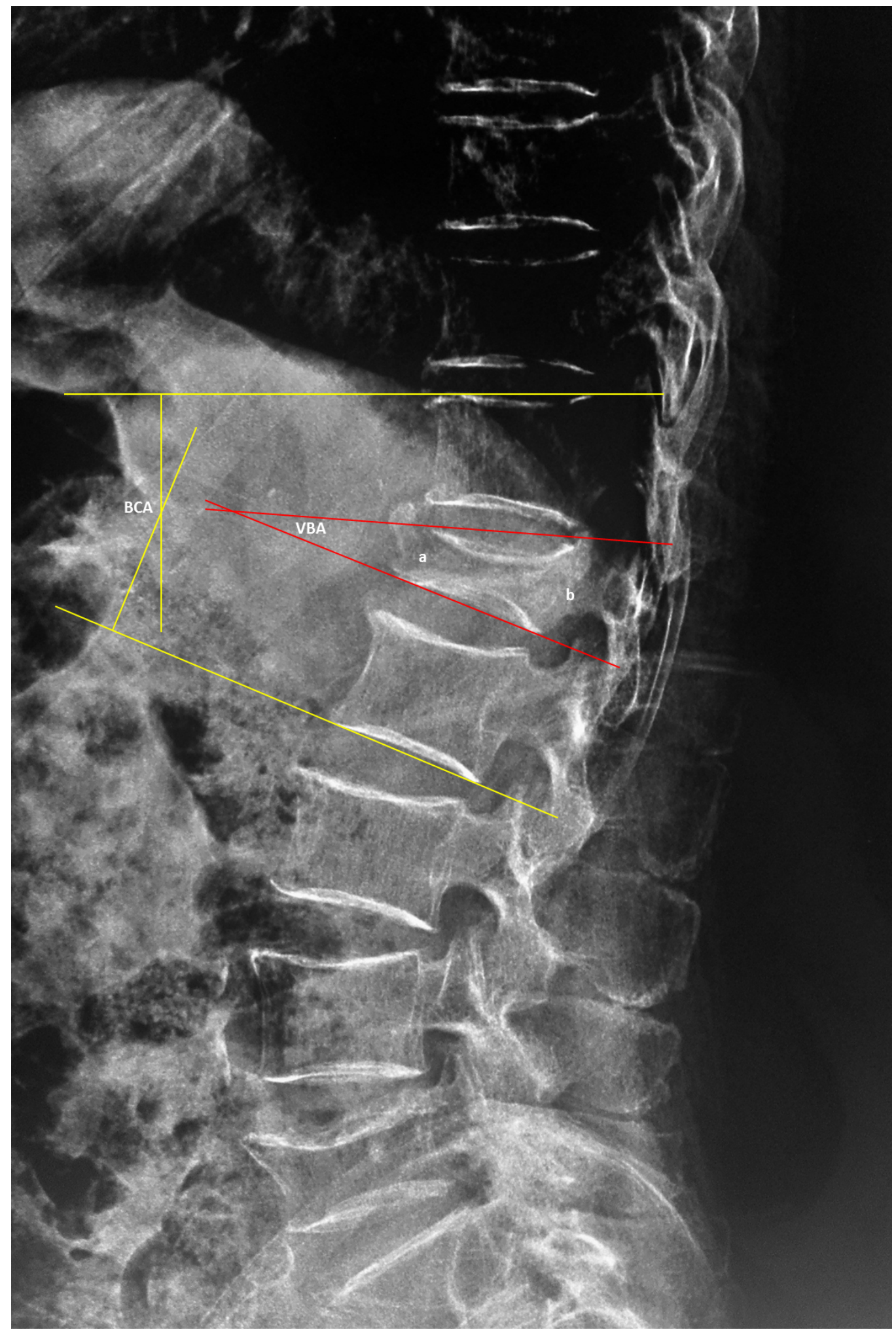

Figure 4 
Schematic diagram of imaging parameter measurement. The measurement methods of VBA and BCA are shown in the figure, and the measurement method of VBI is (a / b) $\times 100 \%$.

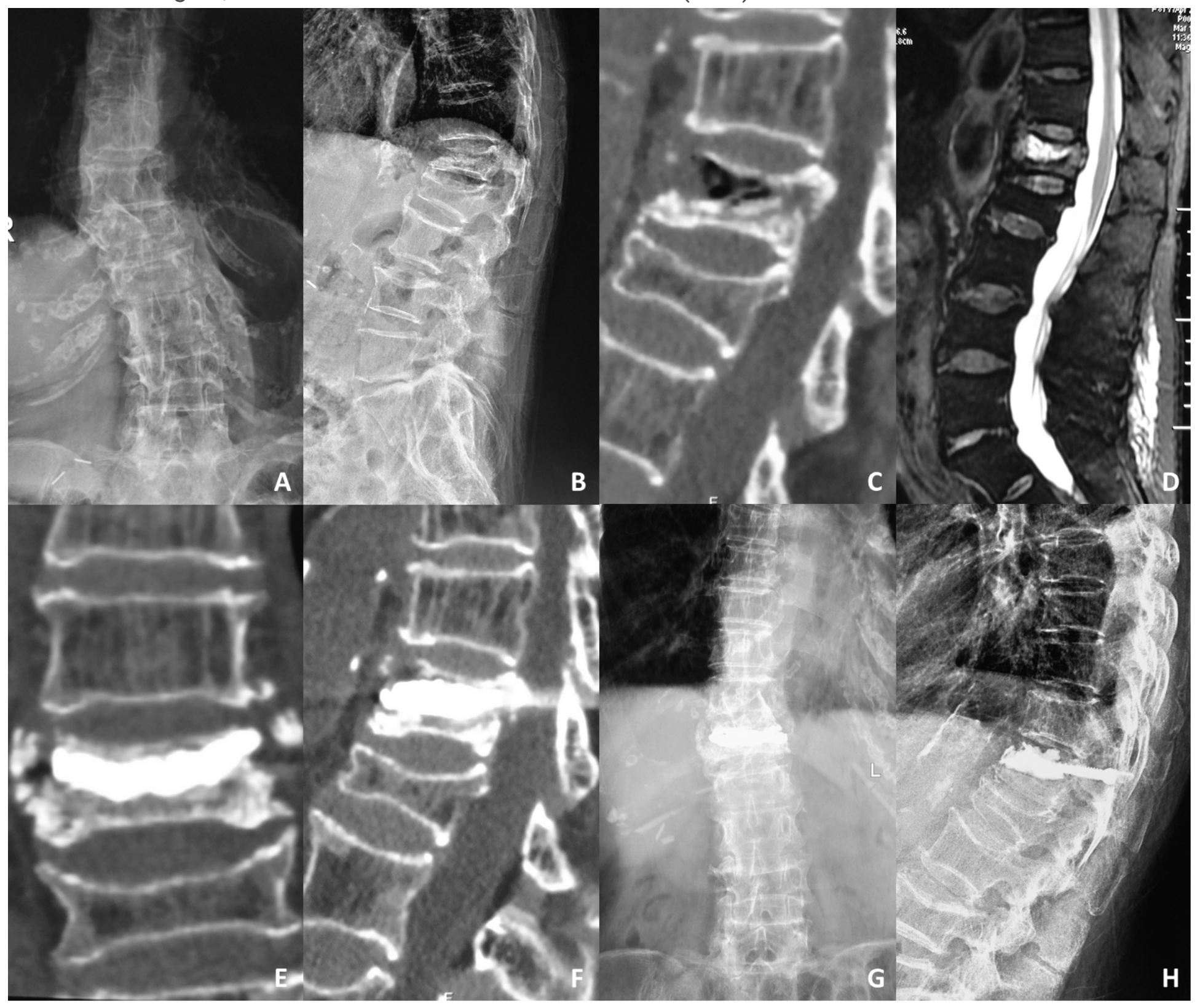

\section{Figure 5}

An 81-year-old female patient, L1 vertebral stage II Kummell disease was treated by new bone cement screw combined with PVP. A and B: Preoperative X-ray showed Kummell disease of L1 vertebra with degenerative scoliosis; her preoperative radiographic parameters were as follows: VBI $33 \%$, VBA $24^{\circ}$, and $\mathrm{BCA} 57^{\circ}$. C: After postural reduction, $\mathrm{CT}$ examination showed that intravertebral vacuum cleft and bone deficiency at vertebral anterior border in L1 vertebrae. D: Preoperative MRI showed that the intravertebral vacuum cleft in L1 vertebrae was liquid signal. E and F: CT examination of 3 days after operation showed that the intravertebral vacuum cleft in L1 was fully filled with bone cement in sagittal and coronal position, the height of vertebrae restored well, and there was no obvious leakage or displacement of bone cement. G and H: At the 3-year follow-up, anteroposterior and lateral X-ray radiographs showed the 
positions of cement and screw were good, and no cement displacement was found; her radiographic parameters were as follows: VBI $73 \%$, VBA $16^{\circ}$, and BCA $38^{\circ}$.

\section{Supplementary Files}

This is a list of supplementary files associated with this preprint. Click to download.

- tables.pdf

- Kummelldiseasedata.xIsx 